\title{
Article \\ Integration of Metabolomic and Clinical Data Improves the Prediction of Intensive Care Unit Length of Stay Following Major Traumatic Injury
}

\author{
Animesh Acharjee ${ }^{1,2,3, *,+} \mathbb{D}$, Jon Hazeldine ${ }^{1,4,+}$, Alina Bazarova ${ }^{2} \mathbb{D}$, Lavanya Deenadayalu ${ }^{2}$, Jinkang Zhang ${ }^{4, \ddagger}$, \\ Conor Bentley ${ }^{1}$, Dominic Russ ${ }^{2}$, Janet M. Lord ${ }^{1,4,5}$, Georgios V. Gkoutos ${ }^{1,2,3} \mathbb{D}$, Stephen P. Young ${ }^{4}$ \\ and Mark A. Foster 1,6
}

\section{check for} updates

Citation: Acharjee, A.; Hazeldine, J.; Bazarova, A.; Deenadayalu, L.; Zhang, J.; Bentley, C.; Russ, D.; Lord, J.M.; Gkoutos, G.V.; Young, S.P.; et al. Integration of Metabolomic and Clinical Data Improves the Prediction of Intensive Care Unit Length of Stay Following Major Traumatic Injury. Metabolites 2022, 12, 29. https:// doi.org/10.3390/metabo12010029

Academic Editor: Masao Kaneki

Received: 7 November 2021 Accepted: 24 December 2021 Published: 31 December 2021

Publisher's Note: MDPI stays neutral with regard to jurisdictional claims in published maps and institutional affiliations.

Copyright: (c) 2021 by the authors. Licensee MDPI, Basel, Switzerland. This article is an open access article distributed under the terms and conditions of the Creative Commons Attribution (CC BY) license (https:/ / creativecommons.org/licenses/by/ $4.0 /)$.
1 Microbiology Research Centre, National Institute for Health Research Surgical Reconstruction, Queen Elizabeth Hospital Birmingham, Birmingham B15 2GW, UK; j.hazeldine@bham.ac.uk (J.H.); conor.bentley@uhb.nhs.uk (C.B.); j.m.lord@bham.ac.uk (J.M.L.); g.gkoutos@bham.ac.uk (G.V.G.); m.foster@bham.ac.uk (M.A.F.)

2 Institute of Cancer and Genomic Sciences, University of Birmingham, Birmingham B15 2TT, UK; albazarova@gmail.com (A.B.); lavanyadeenadayalu@gmail.com (L.D.); drr719@student.bham.ac.uk (D.R.)

3 MRC Health Data Research UK (HDR UK), Midlands Site, Birmingham B15 2TT, UK

4 Institute of Inflammation and Ageing, University of Birmingham, Birmingham B15 2TT, UK; jinkang.zhang@phe.gov.uk (J.Z.); s.p.young@bham.ac.uk (S.P.Y.)

5 National Institute for Health Research Birmingham Biomedical Research Centre, University Hospital Birmingham NHS Foundation Trust, University of Birmingham, Birmingham B15 2GW, UK

6 Royal Centre for Defence Medicine, University Hospital Birmingham, Birmingham B15 2TT, UK

* Correspondence: a.acharjee@bham.ac.uk; Tel.: +44-(0)121-414-7012

+ These authors contributed equally to this work.

$\ddagger$ Current address: Centre for Radiation, Chemicals and Environmental Hazards, UK Health Security Agency, Oxfordshire OX11 0RQ, UK.

Abstract: Recent advances in emergency medicine and the co-ordinated delivery of trauma care mean more critically-injured patients now reach the hospital alive and survive life-saving operations. Indeed, between 2008 and 2017, the odds of surviving a major traumatic injury in the UK increased by nineteen percent. However, the improved survival rates of severely-injured patients have placed an increased burden on the healthcare system, with major trauma a common cause of intensive care unit (ICU) admissions that last $\geq 10$ days. Improved understanding of the factors influencing patient outcomes is now urgently needed. We investigated the serum metabolomic profile of fifty-five major trauma patients across three post-injury phases: acute (days 0-4), intermediate (days 5-14) and late (days 15-112). Using ICU length of stay (LOS) as a clinical outcome, we aimed to determine whether the serum metabolome measured at days 0-4 post-injury for patients with an extended ( $\geq 10$ days) ICU LOS differed from that of patients with a short ( $<10$ days) ICU LOS. In addition, we investigated whether combining metabolomic profiles with clinical scoring systems would generate a variable that would identify patients with an extended ICU LOS with a greater degree of accuracy than models built on either variable alone. The number of metabolites unique to and shared across each time segment varied across acute, intermediate and late segments. A one-way ANOVA revealed the most variation in metabolite levels across the different time-points was for the metabolites lactate, glucose, anserine and 3-hydroxybutyrate. A total of eleven features were selected to differentiate between $<10$ days ICU LOS vs. >10 days ICU LOS. New Injury Severity Score (NISS), testosterone, and the metabolites cadaverine, urea, isoleucine, acetoacetate, dimethyl sulfone, syringate, creatinine, xylitol, and acetone form the integrated biomarker set. Using metabolic enrichment analysis, we found valine, leucine and isoleucine biosynthesis, glutathione metabolism, and glycine, serine and threonine metabolism were the top three pathways differentiating ICU LOS with a $p<0.05$. A combined model of NISS and testosterone and all nine selected metabolites achieved an AUROC of 0.824. Differences exist in the serum metabolome of major trauma patients who subsequently experience a short or prolonged ICU LOS in the acute post-injury setting. Combining metabolomic data with anatomical scoring systems allowed us to discriminate between these two groups with a greater degree of accuracy than that of either variable alone. 
Keywords: metabolomics; omics integration; ICU length of stay; inflammation

\section{Introduction}

Recent advancements in emergency medicine and the co-ordinated delivery of trauma care mean more critically-injured patients now reach the hospital alive and survive lifesaving operations. Indeed, between 2008 and 2017, the odds of surviving a major traumatic injury in the UK increased by nineteen percent [1]. However, the improved survival rates of severely-injured patients have placed an increased burden on the healthcare system, with major trauma a common cause of intensive care unit (ICU) admissions that last $\geq 14$ days [2-4]. Despite representing a small proportion of all hospital admissions, critically-ill patients with a prolonged ICU stay consume the greatest amount of resources on ICU wards [5-7]. Moreover, patients who experience an extended ICU length of stay (LOS) develop more secondary complications [6,8], are at a higher risk of in-hospital mortality [9] and are frequently discharged to rehabilitation centres or skilled nursing facilities [6]. Thus, to allocate resources and improve clinical outcomes, there is an urgent need to develop strategies to identify trauma patients at risk of a prolonged ICU LOS at the earliest opportunity.

Across the settings of traumatic brain injury (TBI), blunt trauma and penetrative injury, prospective and retrospective observational studies have identified several demographic and clinical factors influencing ICU LOS. Falling into one of three distinct categories that relate to (i) pre-trauma parameters, (ii) the traumatic injury itself, or (iii) post-trauma treatment regimens and secondary complications, several factors have been reported to be associated with a prolonged ICU LOS. These factors include male gender [9], increased age $[6,9,10]$, pre-existing co-morbidities [6], higher injury severity $[6,9,10]$, severe head injury [6,10-12], surgical procedures [10] and nosocomial infection $[9,10]$. While an assessment of injury severity at hospital arrival, using either the injury severity score (ISS) or new ISS (NISS), can predict who requires admission to ICU [13-16], these anatomical scoring systems are unable to accurately distinguish between patients who subsequently experience a short or prolonged ICU LOS [17-19], with the area under the receiver operating characteristic (AUROC) curve values ranging from 0.448-0.804 [17-19]. Thus, new models capable of predicting ICU LOS with greater discriminatory power are needed to aid in the development of targeted treatments during the acute post-injury phase.

Metabolomics is a systems-based approach for analysing low molecular weight metabolites present in cells, tissues or biofluids. Applying this analytical technique to serum or plasma samples acquired in the minutes [20], hour [21-23] and days [23-26] following combat injury, TBI or blunt/penetrative trauma has revealed significant traumainduced metabolic perturbations, with changes reported in glucose, fatty acid, amino acid and lipid metabolism. Showing promise as a diagnostic tool for mild TBI [27] and stratifying patients according to injury severity [22,23], metabolic profiling of the circulating metabolome has revealed unique signatures for patients with distinct clinical outcomes. For example, disturbances in amino acid and fatty acid metabolism have been reported in TBI patients who experience post-traumatic cognitive impairments [21], whilst significantly increased concentrations of glucose and glutamate were measured in serum samples obtained at the time of hospital admission from non-survivors of severe trauma [20]. More recently, in a cohort of eighty-six blunt trauma patients, Cyr et al. found that at days 2-5 post-injury, a plasma metabolome enriched in sphingolipids was associated with several improved clinical outcomes, which included a shorter ICU LOS [28]. However, the study did not address whether metabolomic profiles in the immediate post-injury phase could be used to predict which patients would experience an extended LOS in ICU.

Data are emerging to suggest that metabolomic profiling can markedly improve the accuracy of statistical models built on clinical parameters to predict patient outcomes. For example, Oresic and colleagues found that combining data on serum metabolites with 
clinical indices generated a variable that discriminated between TBI patients who reported good and poor outcomes with a level of accuracy that was significantly greater than that of a model based on clinical information alone [22]. Integrating metabolomic and clinical data has also proven helpful in identifying critically-ill patients that are at an increased risk of mortality, with this approach outperforming the predictive abilities of such widely used clinical scoring systems as the sequential organ failure assessment (SOFA) score and the acute physiology and chronic health evaluation II (APACHE II) score [29]. Thus, combining metabolomic profiles with clinical data could be one strategy to improve anatomical-based scoring systems' ability to identify patients at risk of a prolonged ICU LOS.

Here, in this prospective observational study, we investigated the serum metabolomic profile of fifty-five major trauma patients across three post-injury phases: acute (days $0-4)$, intermediate (days 5-14) and late (days 15-112). Using ICU LOS as a clinical outcome, we aimed to determine whether the serum metabolome measured at days $0-4$ post-injury for patients with an extended ( $\geq 10$ days) ICU LOS differed from that of patients with a short ( $<10$ days) ICU LOS. In addition, we investigated whether combining metabolomic profiles with clinical scoring systems would generate a variable that would identify patients with an extended ICU LOS with a greater degree of accuracy than models built on either variable alone.

\section{Methods}

\subsection{Study Design}

This study presents data obtained from subjects enrolled into the Steroids and Immunity from Injury through to Rehabilitation (SIR) study, a prospective cohort study conducted between May 2011 and January 2013 in the Royal Centre for Defence Medicine and Queen Elizabeth Hospital Birmingham, UK. The study protocol was approved by the National Research Ethics Committee South West-Frenchay (Reference-11/SW/0177) and the Ministry of Defence Research Ethics Committee (Reference-116/Gen/10). Details of study inclusion/exclusion criteria and the consenting procedure have been described previously [30].

\subsection{Clinical Data Collection}

Simplified Acute Physiology Score (SAPS), APACHE II, and SOFA scores were calculated daily from the ICU clinical charts while admitted to ICU. Data regarding mortality, ICU and hospital LOS, ISS, NISS and abbreviated injury scale scores were calculated and ratified using the hospital Prescribing Information and Communication System (PICS, University Hospitals Birmingham Foundation Trust), the Trauma Audit Research Network (TARN), a UK-based centralised network that records injury details, or the UK Military equivalent, the Joint Theatre Trauma Registry (JTTR).

\subsection{Clinical Outcomes}

The clinical course of severely injured trauma patients is complicated by persistent inflammation, immunosuppression, and catabolism syndrome (PICS). Patients with an ICU LOS greater than 10 days are more likely to develop PICS that can progress to multiple organ dysfunction syndrome and multiple organ failure [31]. Thus, in this study, we categorised ICU LOS as short ( $<10$ days) or prolonged ( $\geq 10$ days). We provided clinical data in the supplementary Table S1.

\subsection{Blood Sampling and Preparation of Serum}

Blood samples were collected within $24 \mathrm{~h}$ of injury and between 07:30 and 09:00 on days $3,5,10,14,21,28$, and months 2, 3, 4 and 6 post-injury. Samples were collected in BD Vacutainers ${ }^{\circledR}$ (BD Biosciences, Plymouth, UK) containing z-serum clotting activator. After a 30-min incubation at room temperature (RT), samples were centrifuged at $1620 \times g$ for $10 \mathrm{~min}$ at RT. Serum was harvested and stored at $-80^{\circ} \mathrm{C}$ until analysed. 
Comparison of ICU LOS $<10$ days vs. $\geq 10$ days. Unless stated, data are expressed as means with ranges in parentheses. APACHE, Acute physiology chronic health evaluation; GCS, Glasgow coma scale; GSW, Gunshot wound; ICU, Intensive care unit; IED, Improvised explosive device; ISS, Injury severity score; LOS, Length of stay; NISS, New injury severity score; RTC, Road traffic collision; RTS, Revised trauma score; SAPS, Simplified acute physiology score; SOFA, Sequential organ failure assessment score; TRISS, Trauma and injury severity score.

\subsection{Metabolomics Sample Preparation and Analysis}

To remove proteins, which reduce the quality of the NMR spectra, serum was filtered through $3000 \mathrm{MWCO}$ filters [32]. The filtrates were $\mathrm{pH}$ buffered with $50 \mathrm{mM}$ phosphate and made to $10 \%$ with $\mathrm{D}_{2} \mathrm{O}$ and $0.5 \mathrm{mM}$ TMSP (an internal NMR standard). We then assessed the metabolomic profile using NMR spectroscopy as previously described [33,34].

1D 1H NOESY NMR spectra were acquired at $300 \mathrm{~K}$ using a standard 1D-1H-Nuclear Overhauser Effect Spectroscopy pulse sequence with water saturation using pre-sat in a Bruker AVANCE II $600 \mathrm{MHz}$ NMR spectrometer (Bruker Corp., Billerica, MA, USA) equipped with a $1.7 \mathrm{~mm}$ cryoprobe. Spectral width was set to $12 \mathrm{ppm}$, and the scans were repeated 128 times. Spectra were processed using Metabolab within MATLAB (Version 9.2, Mathworks, Cambridge, UK) [35]. Using this package, spectra were phased manually, baseline corrected and referenced to the TMSP resonance $(0.0 \mathrm{ppm})$. To facilitate analysis, each spectrum between 0.2 and $9.0 \mathrm{ppm}$ was reduced in complexity by segmenting into $0.005 \mathrm{ppm}$ bins $(2.5 \mathrm{~Hz})$. Bins between 4.5 and $5.0 \mathrm{ppm}$ containing residual water were removed, and the remaining bins were integrated. Each binned spectrum was normalised using probabilistic quotient normalization [36], transformed using a generalised logarithm (glog) [37] to increase the weighting of smaller peaks. Minor batch anomalies were corrected using the Combat algorithm with the SVA package [38]. Metabolites were identified by comparing spectra with spectra in the human metabolome database [39], using the Chenomx suite of software containing a metabolite library (Chenomx Inc, Edmonton, AB, Canada). Metabolic pathways involved or changes in different subgroups were identified and portrayed using the Metaboanalyst (http:/ / www.metaboanalyst.ca/MetaboAnalyst, accessed on 1 November 2021) version 4.0 software [40].

All metabolites or inflammatory mediators with more than $50 \%$ missing values were excluded from the analysis. Data pre-processing includes logarithmic transformation, centring, and unit variance scaling) was first conducted separately for the metabolomics and other clinical variables and then combined dataset. We provided processed metabolomics data in the supplementary Table S2.

\subsection{Statistical Analysis}

We used univariate ANOVA analysis with post hoc correction $(p<0.05)$ to identify the number of metabolite peaks that were significantly different across different time points. To integrate these with the number of ICU days, we used simple linear regression with a false discovery rate correction (FDR) method (Benjamini-Hochberg Procedure (BH) correction) at $p<0.05$.

\subsection{Partial Least-Squares Discriminant Analysis (PLS-DA) Analysis}

For dichotomous or binary outcomes (ICU LOS $<10$ vs. $\geq 10$ days), we used a supervised classification method called partial least-squares discriminant analysis (PLSDA). We quantified model statistics based on the fraction of the sum of squares for the selected component (R2), which equates to the percentage of the model variance explained, and the predictive ability (Q2). A 10 fold cross-validation was performed to predict and estimate the model performance (whether models were overfitted). In addition to this we have performed a permutation test to derive probability values from the fitted models. We used variable importance in the projection scores (VIP) to prioritize metabolites and clinical features. VIP scores of more than one are considered important, and this score of a 
variable is calculated as a weighted sum of the squared correlations between the PLS-DA components and the original variable. For metabolomics and clinical data, we used a VIP score of more than 1.5 as a cut-off [41]. Further, we used AUC values to provide predictive aspects of the combined markers selected. For analysis, we used the Metaboanalyst version 4.0 software [40] for both model building and VIP score estimation.

\section{Results}

\subsection{Patient Demographics}

A total of 55 male adult trauma patients were enrolled into the study (Table 1). Improvised explosive device was the most common mechanism of injury $(\mathrm{n}=31,56 \%)$ in a cohort whose mean age was 29 years (range, 18-50 years). With a mean ISS of 25.5 (range, 9-75), patients were severely-injured upon hospital arrival and were critically unwell on the day of injury, as demonstrated by mean SOFA and APACHE II scores of 8.3 (range, 0-17) and 20.3 (range, 0-34) respectively (Table 1).

Table 1. Summary of the demographic and clinical characteristics of the study cohort.

\begin{tabular}{|c|c|c|c|c|}
\hline Parameters & $\begin{array}{l}\text { Patients } \\
(\mathrm{N}=55)\end{array}$ & $\begin{array}{c}\text { ICU LOS }<10 \\
\text { Days } \\
(\mathbf{N}=29)\end{array}$ & $\begin{array}{c}\text { ICU LOS } \geq 10 \\
\text { Days } \\
(\mathbf{N}=26)\end{array}$ & $p$-Value \\
\hline Age, years & $29(18-50)$ & $27.5(18-49)$ & $31(21-49.5)$ & 0.17 \\
\hline Male, n (\%) & $55(100)$ & $29(100)$ & $26(100)$ & $\mathrm{N} / \mathrm{A}$ \\
\hline $\begin{array}{c}\text { Mechanism of Injury } \\
\text { Explosive, n (\%) } \\
\text { GSW, n (\%) } \\
\text { Electrical, n (\%) } \\
\text { Blunt, n (\%) } \\
\text { Penetration, n (\%) }\end{array}$ & $\begin{array}{c}31(56.3 \%) \\
8(14.5 \%) \\
1(1.8 \%) \\
13(23.6 \%) \\
2(3.6 \%)\end{array}$ & $\begin{array}{c}16(55.2 \%) \\
5(17.2 \%) \\
0 \\
7(24.1 \%) \\
1(3.5 \%)\end{array}$ & $\begin{array}{c}15(57.7 \%) \\
3(11.5 \%) \\
1(3.8 \%) \\
6(23.1 \%) \\
1(3.9 \%)\end{array}$ & NA \\
\hline ISS & $25.5(9-75)$ & $21(9-38)$ & $30(13-75)$ & 0.005 \\
\hline NISS & $37.3(16-75)$ & $34.4(17-59)$ & $40.6(16-75)$ & 0.07 \\
\hline GCS & $10.3(3-15)$ & $10.7(3-15)$ & $9.9(3-15)$ & 0.61 \\
\hline SOFA (Day 1) & $8.3(0-17)$ & 15 (7.72-17) & $8.6(0-15)$ & 0.41 \\
\hline APACHE II (Day 1) & $20.3(0-34)$ & $17.9(0-29)$ & $22.4(7-34)$ & 0.04 \\
\hline SAPS II (Day 1) & $42.2(0-69)$ & $38.6(11-62)$ & $44.7(0-69)$ & 0.21 \\
\hline TRISS (Day 1) & 77.13 (2.55-99.4) & 78.23 (2.55-99.4) & $75.9(13.27-98.6)$ & 0.76 \\
\hline RTS (Day 1) & $6.3(2.6-7.8)$ & $6.4(2.91-7.84)$ & $6.1(2.63-7.84)$ & 0.55 \\
\hline Ventilator days & $7.8(0-25)$ & $3.3(0-10)$ & $12.9(4-25)$ & $<0.0001$ \\
\hline Operative procedures & $5.8(0-24)$ & $5(0-15)$ & $6.8(0-24)$ & 0.12 \\
\hline ICU LOS & $10(0-34)$ & $4.6(0-9)$ & $16.2(10-33)$ & $<0.0001$ \\
\hline Hospital LOS & $46(7-217)$ & $34(7-72)$ & $59(17-217)$ & 0.02 \\
\hline
\end{tabular}

Patients with an ICU LOS $\geq 10$ days ( $n=26,47 \%$ ) were more severely-injured and critically unwell than patients with an ICU LOS $<10$ days $(n=29,53 \%)$, presenting on the day of injury with significantly higher ISS (30 (range, 13-75) vs. 21 (range, 9-38), $p=0.005$ ) and APACHE II scores (22.4 (range, 7-34) vs. 17.9 (range, $0-29), p=0.04$ ) (Table 1 ). The length of time receiving ventilator support was significantly longer for patients with an ICU LOS $\geq 10$ days (16.2 (range, 10-33) vs. 4.6 (range, $0-9$ ) days, $p=<0.0001$ ) who also had a significantly greater hospital LOS (59 (17-217) vs. $34(7-72)$ days, $p=0.02)$ (Table 1).

\subsection{Changes in the Serum Metabolome over Time}

To understand how the metabolic response to major trauma changed over time, we first compared the serum metabolomes at each of our three post-injury sampling phases. 
Unsupervised PCA (Figure 1A) and supervised PLS-DA (Figure 1B) analysis demonstrated marked variation in the metabolomic profiles measured at the acute, intermediate and late post-injury time-points.

To identify the significant metabolites in each of these segments, a one-way ANOVA was performed with at $p<0.05$, Fisher's LSD corrected. Figure 1C shows the number of metabolites unique to and shared across each time segment. A one-way ANOVA revealed the most variation in metabolite levels across the different time-points was for metabolites putatively identified as, glucose, anserine and 3-hydroxybutyrate respectively. The heatmap presented in the supplementary Figure S1 summarises the changes that occurred in the levels of these metabolites across the three post-injury phases. The percentage of the model variance explained (R2), and the predictive ability (Q2) were found $86 \%$ and $58 \%$ respectively. The PLS model was found statistically significant at $p<0.01$ after permutation test (supplementary Figure S2). Compared to samples acquired in the acute injury setting, significantly lower glucose levels, anserine and 3-hydroxybutyrate were detected at the intermediate and late sampling time-points (Figure 1D). Serum levels of lactate were significantly higher in the late post-injury phase when compared to the intermediate sampling time-point (Figure 1D).

A

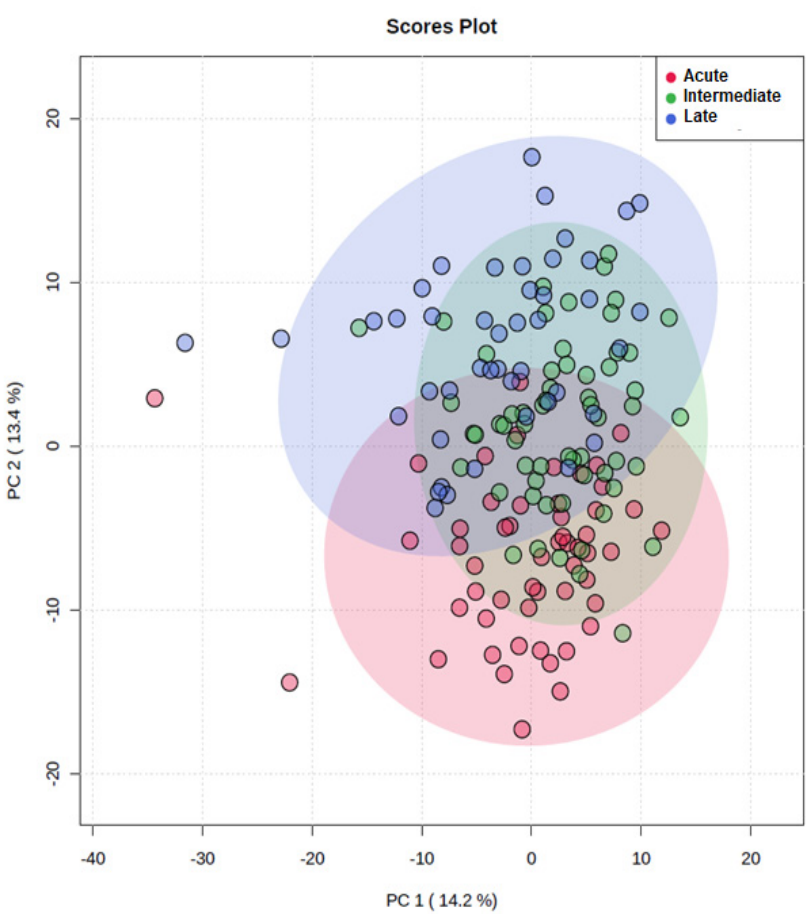

B

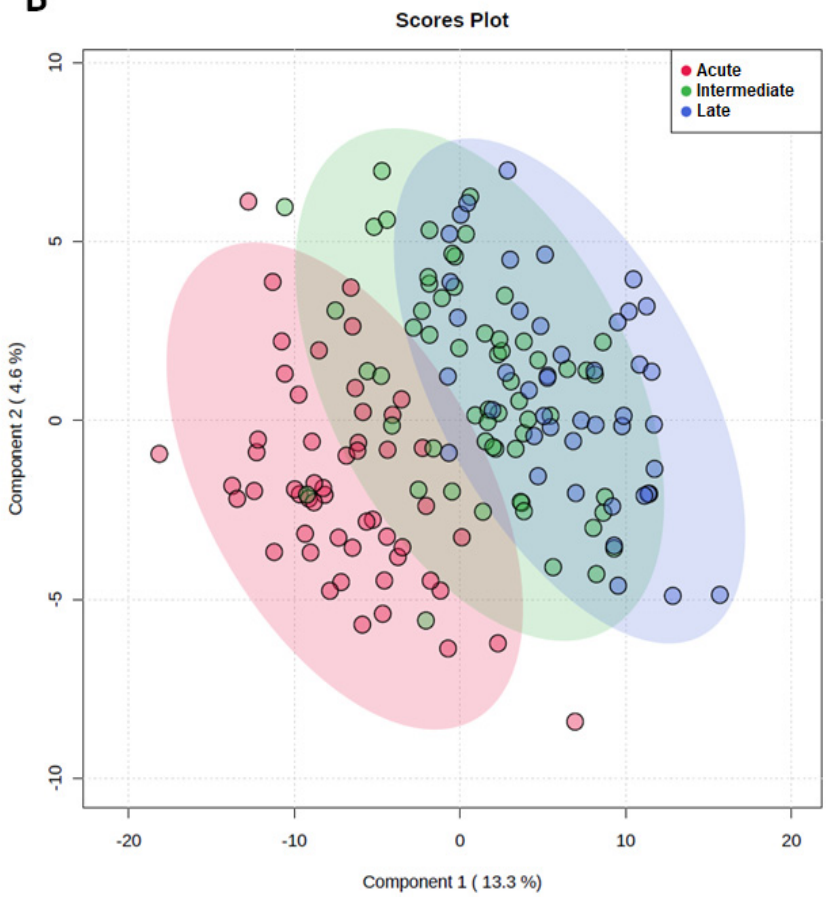

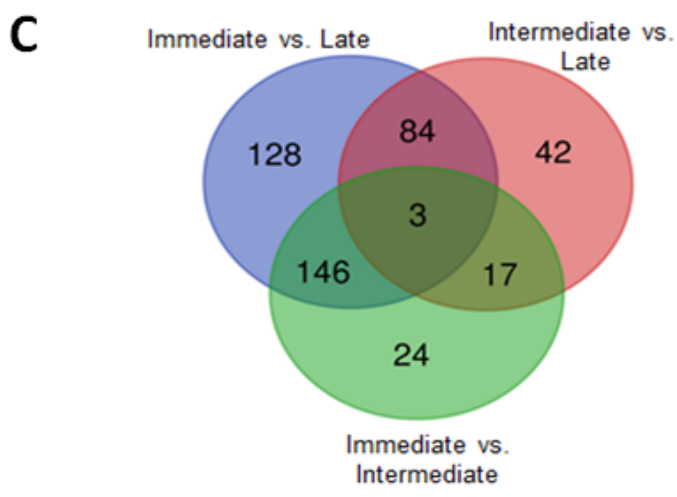

Figure 1. Cont. 


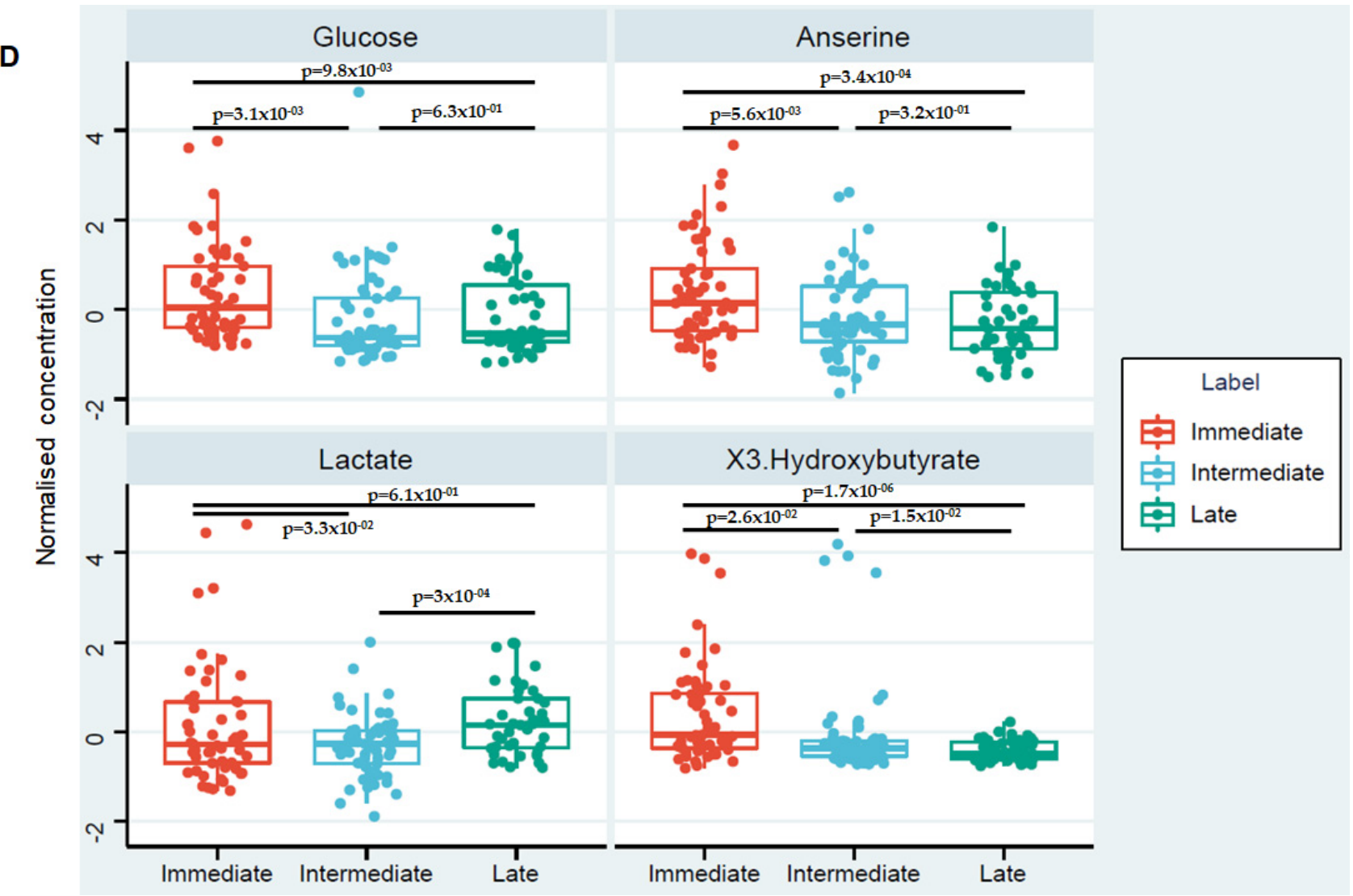

Figure 1. The effect of major traumatic injury on the serum metabolome over time. (A) Unsupervised PCA score plot (B) Supervised PLS-DA score plot analysis of metabolomic data when analysed according to time of sample acquisition. Data are segmented into 3 post-injury phases: acute (red dots), intermediate (green dots) and late (blue dots). Each dot represents a patient. (C) Number of significant metabolites unique to each of the post-injury sampling time-points and the number of common metabolites measured at each time segment. (D) Box plots showing the differences in the levels of metabolites putatively identified as glucose, anserine, lactate and 3-hydroxybutyrate between the three-time segments.

\subsection{Trauma Patients with an ICU LOS $\geq 10$ Days Exhibit a Distinct Metabolomic Profile in the Acute Injury Phase}

PLS-DA models were constructed to investigate whether serum metabolites or clinical variables could be used to discriminate between patients with a short or extended ICU LOS. We aimed to identify potential biomarkers of poor clinical outcome in the acute injury setting. Only metabolomic data derived from the analysis of serum samples acquired in the acute post-injury phase were studied. As shown in (Figure 2A), patients with a prolonged ICU LOS exhibited a distinct metabolomic signature. To identify candidate biomarkers for patients with an ICU LOS $\geq 10$ days, features were ranked based on their Variable Importance in Projection (VIP) score and selected if they had a score of more than 1.5. A total of eleven features were selected; NISS, testosterone, and the metabolites cadaverine, urea, isoleucine, acetoacetate, dimethyl sulfone, syringate, creatinine, xylitol and acetone (Figure 2B). The percentage of the model variance explained (R2), and the predictive ability (Q2) were found 33\% and 29\% respectively. The PLS model was found statistically significant at $p<0.01$ after permutation test (Supplementary Figure S3). Univariate KruskalWallis analysis found that two of these features, namely testosterone and acetoacetate, were significantly different between the two groups, with their levels higher in patients with an ICU LOS $<10$ days (Figure 2C). Comparing the metabolites mentioned above at the 
intermediate and late post-injury phases found no significant differences between the two patient groups.
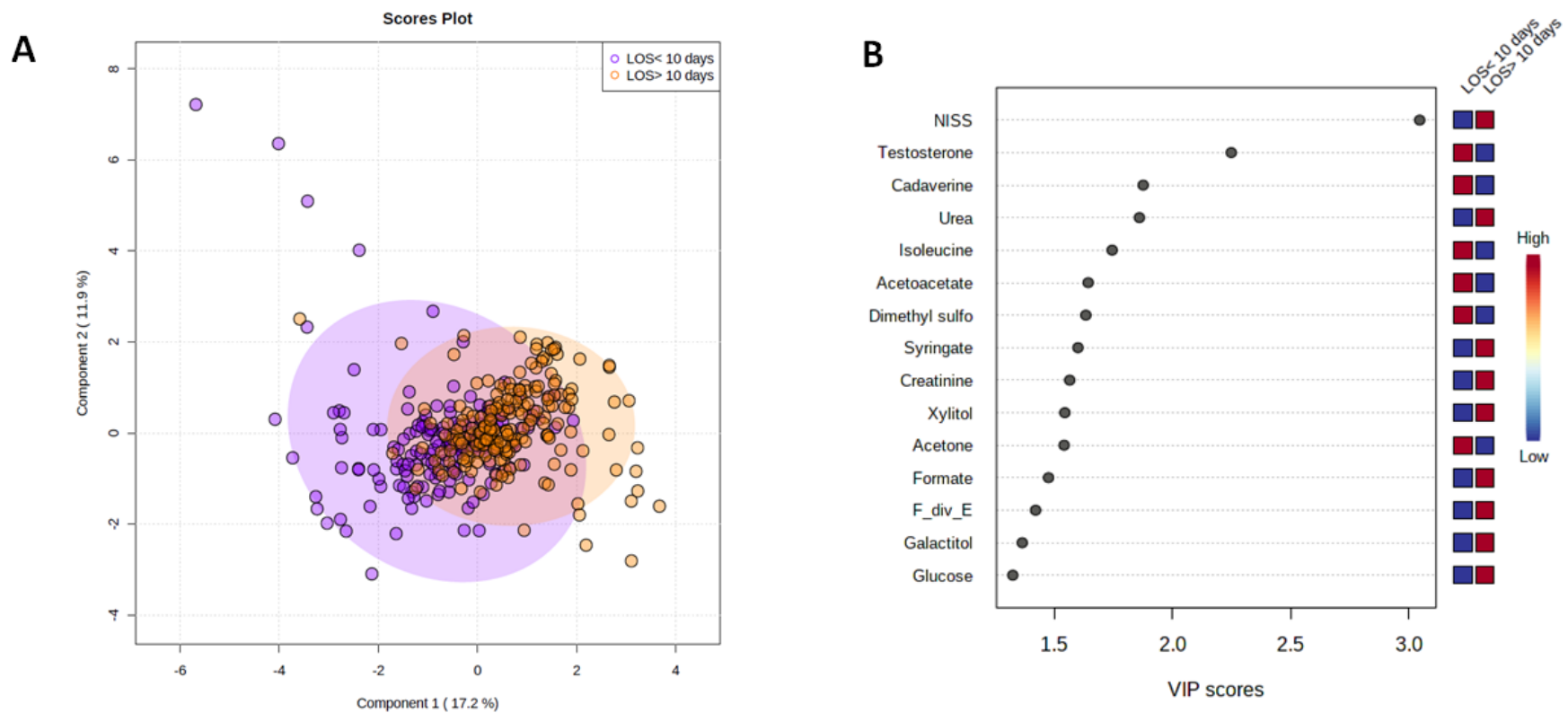

Group 审 LOS $<10$ days

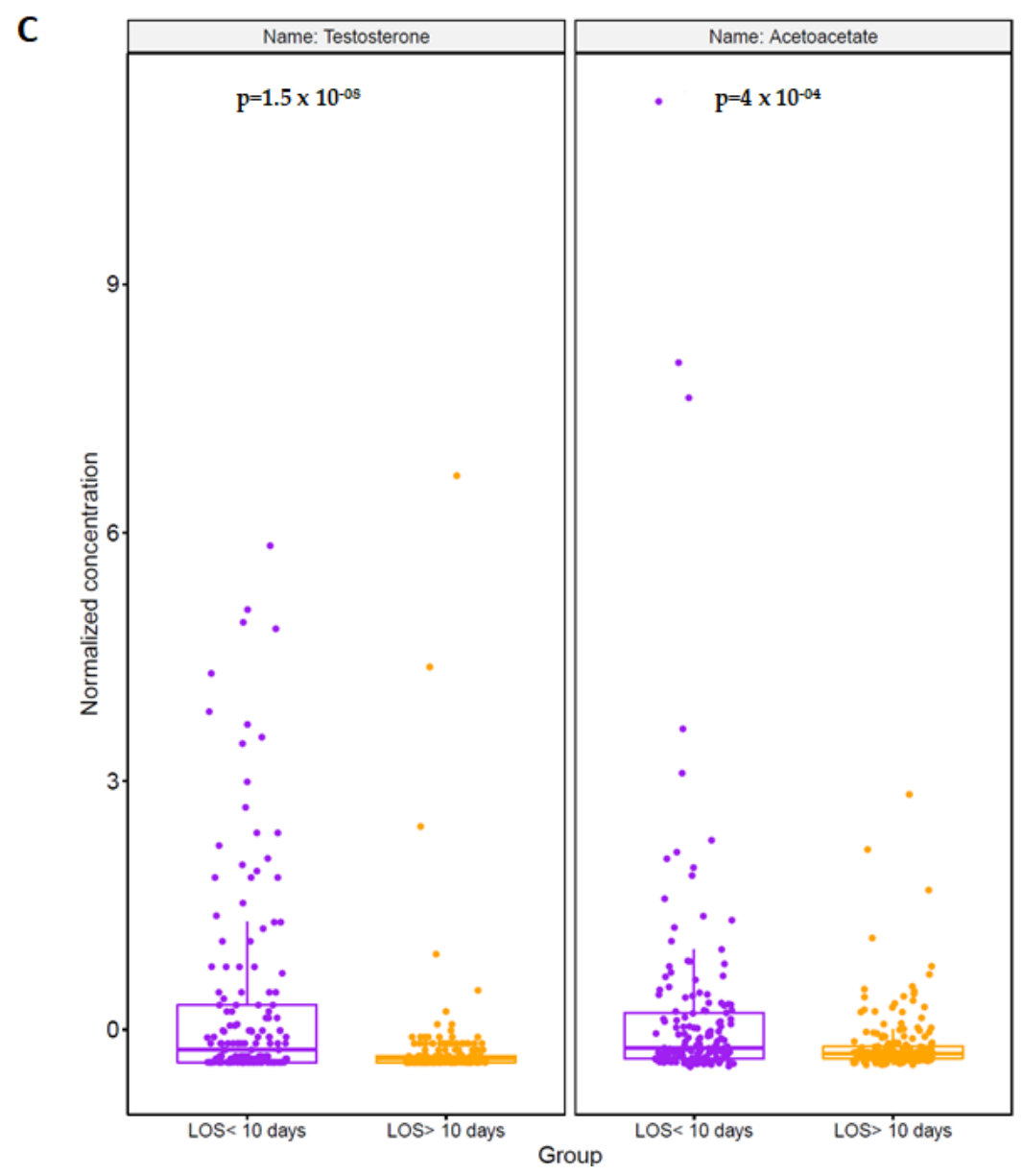

Figure 2. Trauma patients with an ICU LOS $\geq 10$ days present with a distinct serum metabolome 
in the acute post-injury phase. (A) A PLS-DA score plot highlighting the differences in the serum metabolome in the acute post-injury phase of patients with an ICU LOS $<10$ (purple dots) or LOS $\geq 10$ days (orange dots). (B) VIP score plot showing the 11 selected features with a VIP score $>1.5$ that discriminated between ICU patients with a LOS $<10$ or $\geq 10$ days. (C) Comparison of testosterone and acetoacetate levels in serum samples acquired in the acute post-injury phase between patients who experienced an ICU LOS $<10$ or $\geq 10$ days.

\subsection{Clinical Variables and the Acute Metabolic Response Can Discriminate between Patients with} a Short or Extended ICU LOS

To determine whether injury severity, as assessed by the NISS, or metabolites, detected in the acute post-injury phase, could be of prognostic value, we examined the ability of the eleven features selected from our PLS-DA model (Figure 2B) to discriminate between patients with a short or prolonged ICU LOS. The AUROC values for each element are provided in Table 2. As single variables, NISS produced the highest AUROC value of 0.69. A combined model of NISS and testosterone, the two features that demonstrated the greatest discriminatory power as single variables, generated an AUROC of 0.77 (Figure 3A). A model constructed on all nine selected metabolites alone achieved an AUROC of 0.78 , which increased to 0.824 when combined with NISS (Figure 3B).

A

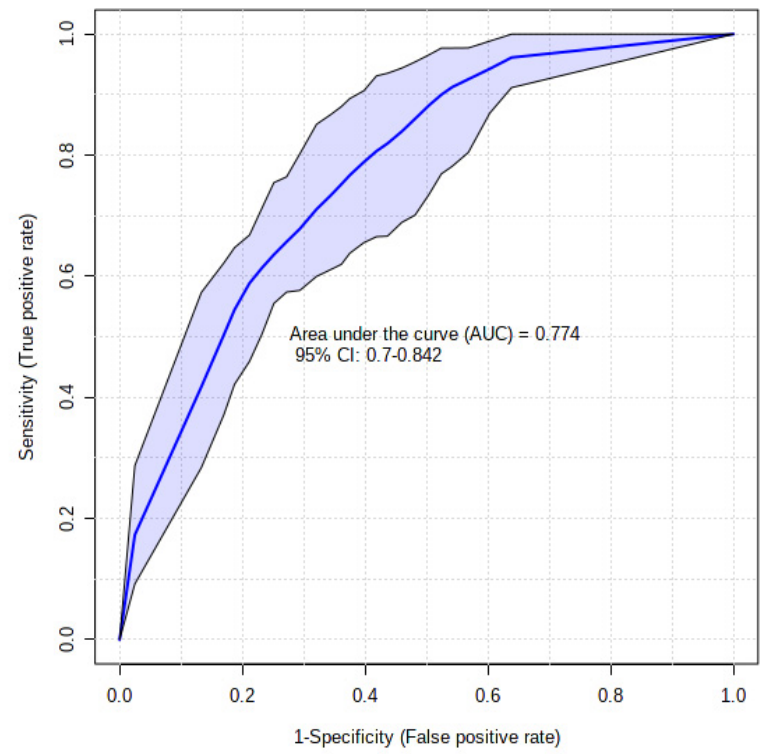

B

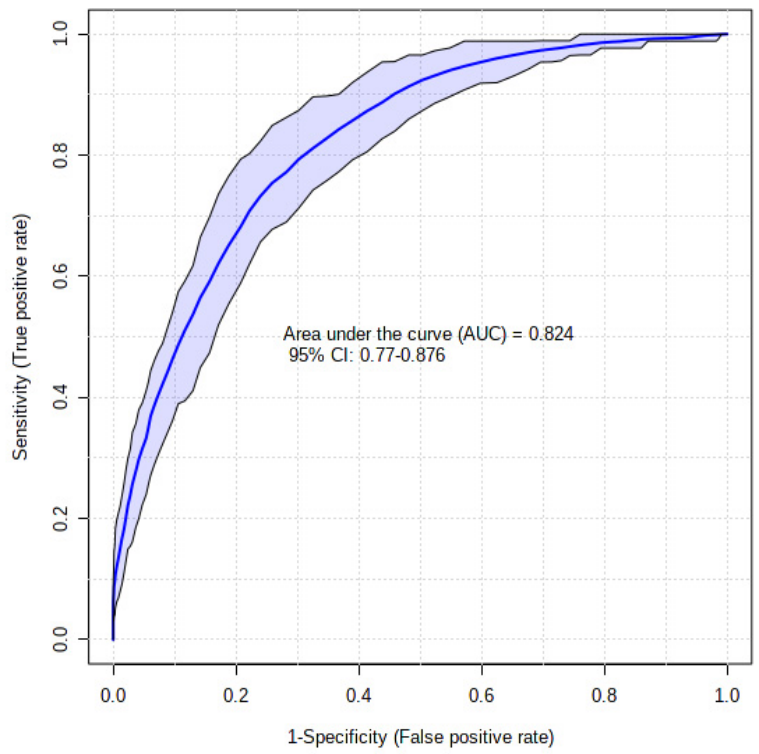

Figure 3. Metabolomic profiling improves the accuracy of clinical variables for discriminating between trauma patients with a short or prolonged ICU LOS. Receiver operator characteristic curves for models built on: (A) the combined variables of NISS and testosterone, or (B) NISS and all 9 serum metabolites (dimethyl sulfone, cadaverine, isoleucine, acetoacetate, urea, syringate, acetone, xylitol and creatinine) detected in the acute post-injury phase that were selected from our PLS-DA model for discriminating between patients with an ICU LOS $<10$ or $\geq 10$ days.

Table 2. Area under the receiver operating characteristic curve (AUROC) values, confidence intervals (C.I) and $p$ values for the metabolites and clinical variables selected from our PLS-DA model based on a VIP $>1.5$.

\begin{tabular}{cccc}
\hline Feature & AUROC & Confidence Interval & $p$ Value \\
\hline NISS & 0.69434 & $0.639-0.748$ & $2.62 \times 10^{-12}$ \\
\hline Testosterone & 0.668566 & $0.612-0.728$ & $3.27 \times 10^{-7}$ \\
\hline Dimethyl sulfone & 0.636886 & $0.577-0.694$ & 0.000234 \\
\hline
\end{tabular}


Table 2. Cont.

\begin{tabular}{cccc}
\hline Feature & AUROC & Confidence Interval & $p$ Value \\
\hline Cadaverine & 0.628626 & $0.55-0.682$ & $2.25 \times 10^{-5}$ \\
\hline Isoleucine & 0.625872 & $0.568-0.68$ & $8.30 \times 10^{-5}$ \\
\hline Acetoacetate & 0.609176 & $0.558-0.675$ & 0.000214 \\
\hline Urea & 0.595153 & $0.536-0.652$ & $2.64 \times 10^{-5}$ \\
\hline Syringate & 0.591759 & $0.537-0.645$ & 0.000314 \\
\hline Acetone & 0.589022 & $0.434-0.621$ & 0.000523 \\
\hline Xylitol & 0.583723 & $0.522-0.64$ & 0.000509 \\
\hline Creatinine & 0.552331 & $0.512-0.612$ & 0.000426 \\
\hline
\end{tabular}

3.5. Altered Amino Acid Metabolism Is a Feature of the Acute Metabolic Response for Patients with a Prolonged ICU LOS

To identify the metabolic pathways associated with the selected metabolites, and thus an ICU LOS $\geq 10$ days, enrichment analysis using MetaboAnalyst was performed. We found valine, leucine and isoleucine biosynthesis, glutathione metabolism, and glycine, serine and threonine metabolism were the top three pathways with a $p<0.05$ (Figure 4).

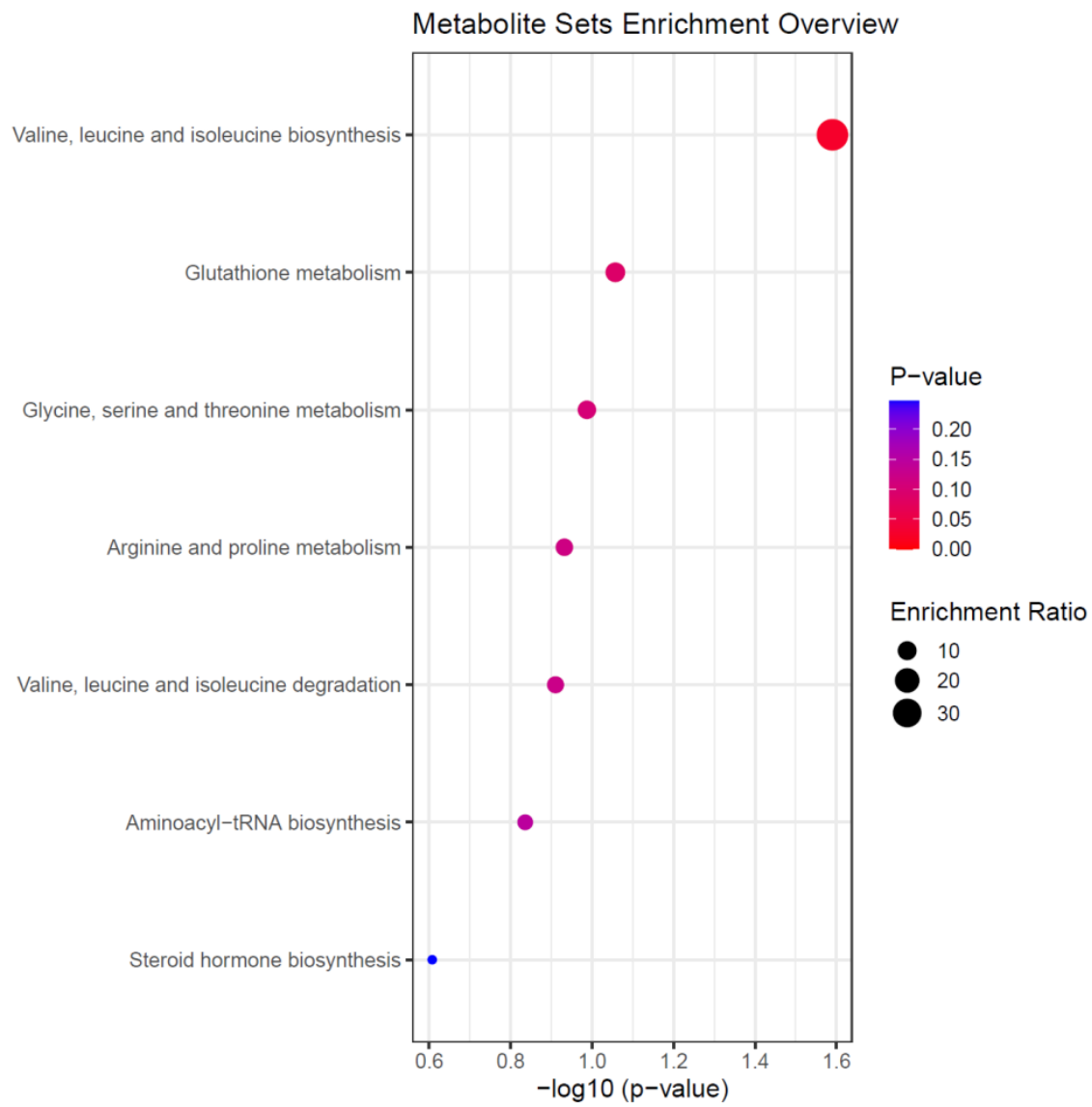

Figure 4. Summary of the altered metabolic pathways in patients with an ICU LOS $\geq 10$ days. Data were derived from enrichment analysis of the nine selected metabolites from our PLS-DA model for discriminating between patients with an ICU LOS $<10$ or $\geq 10$ days. Enrichment Ratio is computed by Hits $/$ Expected, where hits = observed hits; expected $=$ expected hit. 


\section{Discussion}

Ranging from an increased incidence of secondary complications to discharge to specialised care facilities, critically ill patients with an extended ICU LOS experience poor short and long-term clinical outcomes [6,8]. In paediatric and adult trauma settings, several groups have investigated whether an assessment of injury severity at hospital presentation can aid in the identification of patients at risk of a prolonged ICU LOS [17-19,42]. Whilst some studies have reported either no or weak positive associations between injury severity scores and ICU LOS [42], others have shown that statistical models built on the anatomical scoring systems of ISS or NISS can distinguish between patients who experience a short or long ICU LOS [17-19]. However, the predictive ability of these models range from poor to fair, meaning there is a need to develop new models with greater discriminative power. Here, we have analysed the serum metabolome of fifty-five severely injured male trauma patients, describing significant changes across time in metabolic pathways related to carbohydrate, amino acid and fatty acid metabolism. Importantly, analysis of samples collected in the acute post-injury phase revealed distinct metabolomic profiles for patients who subsequently experienced a prolonged ICU LOS. To predict an ICU LOS $\geq 10$ days, a combined anatomical-physiological model built on nine serum metabolites and NISS outperformed models designed on either variable alone. Thus, akin to findings reported in other critical care settings [29], our data demonstrate the importance of studying the physiological response to major trauma when identifying patients at risk of poor clinical outcomes.

Our current understanding of the metabolic response to traumatic injury is based almost entirely upon the results of studies that have analysed serum samples acquired from patients at either a single post-injury time-point $[20-22,24,43]$ or at multiple timepoints during the acute injury phase (days $1-7)[23,25,26,28,44,45]$. Thus, via the analysis of blood samples obtained in the acute (days 0-4), intermediate (days 5-14) and late (days 15-112) post-injury phases, our study has provided novel insights into both the kinetics of the metabolic response to injury as well as the long-term metabolomic profiles of major trauma patients. Revealing time-associated alterations in lipid, protein and carbohydrate metabolism, we detected significantly lower levels of glucose, anserine and 3-hydroxybutyrate in serum samples acquired from patients at our intermediate and late post-injury time-points when compared to the levels present during the acute sampling phase. Major trauma patients presenting with hyperglycaemia and elevated ketone bodies at hospital admission have been reported previously [20,23]. Mechanistically, these early perturbations in carbohydrate and fatty acid metabolism are likely to be driven by immediate trauma-induced changes in the circulating hormonal milieu. For instance, catecholamines, glucocorticoids, growth hormone and/or glucagon, whose levels are all elevated post-injury, promote peripheral insulin resistance, reduce muscle glucose uptake and trigger hepatic glycogenolysis, gluconeogenesis, and ketogenesis [46-50]. In the only other study to our knowledge to have prospectively studied the circulating metabolome of injured military personnel, Lusczek et al. analysed serial plasma samples from seventyeight combat causalities in the acute injury phase $(0-24 \mathrm{~h})$. In line with the changes we observed in carbohydrate metabolism over time, the authors detected lower glucose concentrations 8 and $24 \mathrm{~h}$ post-injury compared to the levels they measured in samples acquired at hospital admission [23]. However, in contrast to our observations, the same study found concentrations of 3-hydroxybutyrate increased in the circulation of major trauma patients over time [23]. This difference may reflect our different sampling times or that glucose levels were sufficiently low in their patient cohort to require an increase in ketoacidosis.

Across time windows of $0-24$ and $0-120 \mathrm{~h}$, longitudinal studies of blunt trauma patients and combat casualties have reported a time associated decrease in the plasma levels of lactate $[23,28]$. In contrast to these observations, we found lactate levels were significantly higher in the serum samples we acquired from patients at our late stage sampling time-point when compared to the intermediate phase. Based on the findings of the above-mentioned studies, our observation of hyperlactaemia in trauma patients 
sampled 15-112 days post-injury is surprising. The differences observed in our study could be attributed to the high number of severely injured patients that remained on ICU with ongoing multiple organ dysfunction/failure. Early resuscitation, restoring tissue perfusion will clear lactate from the circulation and is indeed used as a surrogate measure of resuscitation success. While ongoing multi-organ dysfunction and sepsis will generate more lactate, and so it is unreliable [51,52].

In civilian and military trauma settings, distinct metabolic phenotypes have been described for patients who experience poor clinical outcomes. Adding to a body of literature that has demonstrated distinct metabolomic profiles for major trauma patients who: (i) succumb to their injuries $[20,23,24,45,53]$ (ii) require extended ventilator use [28] or (iii) develop nosocomial infections that progress to sepsis, [28,54] we have shown that the early metabolic response to injury differs between patients who experience a short or extended ICU LOS. Analysing samples acquired in the acute post-injury phase, we detected a distinct metabolomic signature for patients with a prolonged ICU LOS, with VIP scores revealing that testosterone and nine metabolites (cadaverine, urea, isoleucine, acetoacetate, dimethyl sulfone, syringate, creatinine, xylitol and acetone) made the greatest contributions to PLS-DA models that distinguished between trauma patients with an ICU LOS $<10$ or $\geq 10$ days. Of these selected features, testosterone levels and the ketone body acetoacetate were significantly higher in patients with an ICU LOS $<10$ days.

Our observation that hypogonadism is associated with poor clinical outcomes posttrauma agrees with the results of previous prospective studies of critically ill patients that found low testosterone levels were associated with and/or predictive of mortality, increased ventilator use, a prolonged ICU LOS and cognitive/functional decline [55-58]. Although evident within hours of injury and persisting for up to two months [30], how a trauma-induced reduction in testosterone levels influences the physiological response to injury in humans is poorly understood [59]. The central stimulation of the hypothalamic pituitary adrenal axis, unhindered by the suppression of the gonadal axis, has been well reported, but how much this is due to design or consequence is unknown [59]. Given that testosterone is important for wound healing and the maintenance of bone density and muscle mass, we speculate that hypogonadism post-trauma contributes to a state of systemic catabolism and promotes poor wound healing that leads to an extended ICU LOS [60]. In support of this idea, we have previously shown that the gradual recovery in gonadal androgen production post-injury coincides with a transition from catabolism to anabolism, as assessed by urinary nitrogen excretion and biceps brachii muscle thickness [30]. Thus, could androgen supplementation be used to aid clinical recovery and reduce ICU LOS post-trauma? Widely studied in the setting of thermal injury, treatment with the synthetic androgen oxandrolone has been shown to improve donor-site healing time and reduce net weight and lean body mass loss in patients with severe burns [61]. Associated with a shorter hospital LOS, these clinical benefits highlight the potential therapeutic value of androgen supplementation [61]. That said, whether such benefits extend to major trauma patients is currently unclear. For example, whilst reduced nitrogen and/or 3-methylhistidine excretion has been reported for polytrauma and TBI patients treated with the testosterone analogue nandrolone [62,63], oxandrolone therapy in trauma patients resulted in prolonged ventilator use and a longer ICU LOS [64]. As these studies recruited a relatively small number of patients with different mechanisms of injury and varied not only in the type of nutritional support they provided to patients but also in the dose and timing of steroid therapy, further studies are needed to establish whether treatment with anabolic steroids can improve the clinical outcomes of major trauma patients.

A product of fatty acid metabolism, ketone bodies increase cerebral blood flow and protect neuronal cells from stress and cytotoxicity-induced apoptosis [65]. However, as patients who had TBI as a significant component of their injury were excluded from our study, it is unlikely that these neuroprotective effects contributed to the association we observed between raised acetoacetate levels and a reduced ICU LOS. Aside from its cerebral benefits, acetoacetate, via activation of mitogen-activated protein kinase signalling, acceler- 
ates muscle cell proliferation and regeneration [66]. Moreover, in the mitochondrial matrix, acetoacetate can be reduced to 3-hydroxybutyrate, reducing oxidative stress and blunt pro-inflammatory cytokine responses [67]. Thus, these anti-catabolic and anti-inflammatory properties provide potential mechanistic explanations for the improved clinical outcomes we observed for patients with elevated circulating levels of acetoacetate post-injury.

Previous studies have suggested that a patient's metabolic response to trauma impacts upon their ICU LOS. Associated with significantly lower levels of circulating proinflammatory mediators, Cyr et al. found that patients who presented 2-5 days post-injury with a plasma metabolome enriched in sphingolipids had a shorter ICU LOS than patients who exhibited no time-dependent change in sphingolipid levels [28]. Conversely, increased levels of metabolites related to amino acid and glucose metabolism were measured in plasma samples acquired 12-24 h post-injury from blunt and penetrating trauma patients who experienced an ICU LOS $\geq 14$ days compared to those who recovered rapidly (ICU LOS $\leq 7$ days) [44]. However, no study to our knowledge had investigated whether metabolic profiles could be used to predict a patient's ICU LOS. For discriminating between patients with an ICU LOS $<10$ or $\geq 10$ days, testosterone and the nine metabolites selected from our PLS-DA plots were generated as single variables, AUROC values lower than those derived from a model built on a patients NISS. However, a model constructed on all nine metabolites provided greater discriminatory power than NISS alone. The predictive ability of the model, Q2 was low which indicates that ICU LOS is a complex clinical measure and very few metabolites impacting the outcome variable. That model built on combinations of metabolites outperform those designed on single metabolites for predicting patient outcome has been demonstrated previously. For example, Servia and colleagues reported that combined measurement of cortisol and myristic acid discriminated between survivors and non-survivors of traumatic injury with greater accuracy than a panel of six individual metabolites [24]. With an AUROC value of 0.824 , our best model for distinguishing between patients with an ICU LOS $<10$ or $\geq 10$ days was built upon combining our nine selected metabolites and a NISS and gives an 18\% improvement over NISS alone. However, NISS still acts as one of the important predictors to discriminate short ( $<10$ days) vs. long ( $>10$ days) ICU LOS. Highlighting the benefits of studying the physiological response to injury, this observation adds to data derived from other studies of critically-ill patients where merging clinical and metabolomic data improved the accuracy of models designed to predict poor outcome following TBI22 or mortality risk in septic patients [29]. With technological advancements now making it possible to process metabolomic data within 3-h of sample analysis [68], it is conceivable that clinicians could gain access to the metabolic profiles of their patients in the acute injury phase. Our study suggests that such data, when used alongside anatomical scoring systems, could be used to assist in the design of personalised treatment and/or management protocols for patients at risk of poor clinical outcomes.

This study has several limitations. Our findings require validation in larger independent cohorts by enrolling a relatively small number of patients into a study conducted at a single major trauma centre. Related to this, our analyses were performed on samples acquired only from male trauma patients of young to middle age. Thus, whilst beneficial in reducing the confounding effects that age and sex have on the metabolic response to traumatic injury [25], this patient demographic means our results may not be generalizable to other trauma patients. The generalisability of our findings may also be hampered by our recruitment of both civilian and military trauma patients who presented with diverse patterns and severities of injury. However, it should be noted that for our assessment of whether metabolomic profiles could be used to discriminate between patients according to their ICU LOS, no significant differences were detected in the mechanisms of injury with an ICU LOS $<10$ or $\geq 10$ days. The most important limitation of our study was the absence of a healthy control (HC) cohort, which prevented us from reporting upon both the short and long-term effects of major trauma on the serum metabolome. Previous trauma-based studies lacking a HC cohort have used metabolomic data obtained from independent 
studies as reference values for comparison [28]. The inclusion of military personnel within our trauma cohort prevented us from adopting this approach as military training alone results in distinct metabolomic signatures $[69,70]$.

\section{Conclusions}

In summary, we have shown that differences exist in the serum metabolome of major trauma patients who subsequently experience a short or prolonged ICU LOS in the acute post-injury setting. Combining metabolomic data with anatomical scoring systems allowed us to discriminate between these two groups with a greater degree of accuracy than that of either variable alone. When considered alongside the results of studies that have reported distinct genomic signatures for trauma patients who develop multiple organ failure and nosocomial infections [71,72], our data highlights the benefits offered by "omics" research for aiding in the identification of patients at risk of poor clinical outcome following major traumatic injury.

Supplementary Materials: The following supporting information can be downloaded at: https:// www.mdpi.com/article/10.3390/metabo12010029/s1, Figure S1: Heatmap of the selected metabolites across multiple patients; Figure S2: Permutation test statistics over 100 iterations across multiple segments; Figure S3: Permutation test statistics over 100 iterations with selected metabolites for ICU LOS > 10 vs. <10; Table S1: List of the clinical parameters; Table S2: List of the selected metabolite data set.

Author Contributions: Conceptualization, A.A. and M.A.F.; Data curation, A.A., L.D., J.Z., C.B., S.P.Y. and M.A.F.; Formal analysis, A.A., J.H., A.B., L.D., J.Z. and D.R.; Funding acquisition, G.V.G. and S.P.Y.; Investigation, A.A., J.H., A.B., L.D., J.Z., C.B., J.M.L., G.V.G., S.P.Y. and M.A.F.; Methodology, A.A., A.B. and M.A.F.; Project administration, A.A. and G.V.G.; Software, A.A., A.B. and S.P.Y.; Supervision, J.M.L. and M.A.F.; Validation, A.A. and J.H.; Visualization, A.A.; Writing-original draft, J.H., A.B., L.D., C.B., D.R., J.M.L., G.V.G., S.P.Y. and M.A.F.; Writing-review \& editing, A.A., J.H., A.B., L.D., J.Z., C.B., D.R., J.M.L., G.V.G., S.P.Y. and M.A.F. All authors have read and agreed to the published version of the manuscript.

Funding: The SIR Study was part of the Surgeon General's Casualty Nutrition Study (SGCNS), a Ministry of Defence funded project. The detailed ongoing analysis of the cohort was funded by the National Institute for Health Research (NIHR) Surgical Reconstruction and Microbiology Research Centre (SRMRC), Birmingham. JH is supported by the National Institute for Health Research (NIHR) Surgical Reconstruction and Microbiology Research Centre (SRMRC). GVG also acknowledges support from H2020-EINFRA (731075) and the National Science Foundation (IOS:1340112) as well as support from the NIHR Birmingham ECMC, the NIHR Birmingham Biomedical Research Centre and the MRC HDR UK. The views expressed in this publication are those of the authors and not necessarily those of the NHS, the National Institute for Health Research, the Medical Research Council or the Department of Health, UK. The funders provided the financial support to this research but had no role in the design of the study, analysis, interpretations of the data and in writing the manuscript.

Institutional Review Board Statement: The protocol was ethically approved by the NRES Committee South West-Frenchay 11/SW/0177 and MOD REC 116/Gen/10.

Informed Consent Statement: All patients were consented when entered into the trial. When an injured patient lacked capacity under Section 3, the study team would gain advice from a 'consultee', personal or otherwise before entry into the study (under the terms of Section 32 and Section 33 of the Mental Capacity Act 2005). When an individual regained capacity, full informed consent was obtained from the patient before they continued in the study.

Data Availability Statement: Data can be found in the supplementary files or https://figshare.com/ account/home\#/projects/129278.

Conflicts of Interest: The authors declare no conflict of interest.

\section{References}

1. Moran, C.G.; Lecky, F.; Bouamra, O.; Lawrence, T.; Edwards, A.; Woodford, M.; Willett, K.; Coats, T.J. Changing the System-Major Trauma Patients and Their Outcomes in the NHS (England) 2008-2017. EClinicalMedicine 2018, 2-3, 13-21. [CrossRef] 
2. Wong, D.T.; Gomez, M.; McGuire, G.P.; Kavanagh, B. Utilization of intensive care unit days in a Canadian medical-surgical intensive care unit. Crit. Care Med. 1999, 27, 1319-1324. [CrossRef] [PubMed]

3. Wunsch, H.; Gershengorn, H.; Scales, D.C. Economics of ICU Organization and Management. Crit. Care Clin. 2012, 28, 25-37. [CrossRef]

4. Pastores, S.M.; Dakwar, J.; Halpern, N.A. Costs of Critical Care Medicine. Crit. Care Clin. 2012, 2, 1-10. [CrossRef]

5. Suistomaa, M.; Niskanen, M.; Kari, A.; Hynynen, M.; Takala, J. Customised prediction models based on APACHE II and SAPS II scores in patients with prolonged length of stay in the ICU. Intensive Care Med. 2002, 28, 479-485. [CrossRef]

6. Ong, A.W.; Omert, L.A.; Vido, D.; Goodman, B.M.; Protetch, J.; Rodriguez, A.; Jeremitsky, E. Characteristics and outcomes of trauma patients with ICU lengths of stay 30 days and greater: A seven-year retrospective study. Crit. Care 2009, 13, R154. [CrossRef]

7. Halpern, N.A.; Pastores, S.M. Critical care medicine in the United States 2000-2005: An analysis of bed numbers, occupancy rates, payer mix, and costs. Crit. Care Med. 2010, 38, 65-71. [CrossRef] [PubMed]

8. Vincent, J.L.; Rello, J.; Marshall, J.; Silva, E.; Anzueto, A.; Martin, C.D.; Moreno, R.; Lipman, J.; Gomersall, C.; Sakr, Y.; et al. International study of the prevalence and outcomes of infection in intensive care units. JAMA-J. Am. Med. Assoc. 2009, 302, 2323-2329. [CrossRef]

9. Kashkooe, A.; Yadollahi, M.; Pazhuheian, F. What factors affect length of hospital stay among trauma patients? A single-center study, Southwestern Iran. Chin. J. Traumatol. 2020, 23, 176-180. [CrossRef] [PubMed]

10. Böhmer, A.B.; Just, K.S.; Lefering, R.; Paffrath, T.; Bouillon, B.; Joppich, R.; Wappler, F.; Gerbershagen, M.U. Factors influencing lengths of stay in the intensive care unit for surviving trauma patients: A retrospective analysis of 30,157 cases. Crit. Care 2014, 18, R143. [CrossRef]

11. Lazaridis, C.; Yang, M.; DeSantis, S.M.; Luo, S.T.; Robertson, C.S. Predictors of intensive care unit length of stay and intracranial pressure in severe traumatic brain injury. J. Crit. Care 2015, 30, 1258-1262. [CrossRef]

12. Chalya, P.L.; Gilyoma, J.M.; Dass, R.M.; Mchembe, M.D.; Matasha, M.; Mabula, J.B.; Mbelenge, N.; Mahalu, W. Trauma admissions to the Intensive care unit at a reference hospital in Northwestern Tanzania. Scand. J. Trauma Resusc. Emerg. Med. 2011, 19, 61. [CrossRef] [PubMed]

13. Lavoie, A.; Moore, L.; LeSage, N.; Liberman, M.; Sampalis, J.S. The Injury Severity Score or the New Injury Severity Score for predicting intensive care unit admission and hospital length of stay? Injury 2005, 36, 477-483. [CrossRef]

14. Balogh, Z.J.; Varga, E.; Tomka, J.; Süveges, G.; Tóth, L.; Simonka, J.A. The new injury severity score is a better predictor of extended hospitalisation and intensive care unit admission than the injury severity score in patients with multiple orthopaedic injuries. J. Orthop. Trauma 2003, 17, 508-512. [CrossRef]

15. Tamim, H.; Al Hazzouri, A.Z.; Mahfoud, Z.; Atoui, M.; El-Chemaly, S. The injury severity score or the new injury severity score for predicting mortality, intensive care unit admission and length of hospital stay: Experience from a university hospital in a developing country. Injury 2008, 39, 115-120. [CrossRef]

16. Kahloul, M.; Bouida, W.; Boubaker, H.; Toumi, S.; Grissa, M.H.; Jaafar, A.; Louzi, M.; Boukef, R.; Gahbiche, M.; Nouira, S. Value of anatomic and physiologic scoring systems in outcome prediction of trauma patients. Eur. J. Emerg. Med. 2014, 21, 125-129. [CrossRef]

17. Li, H.; Ma, Y.F. New injury severity score (NISS) outperforms injury severity score (ISS) in the evaluation of severe blunt trauma patients. Chin. J. Traumatol. 2021, 24, 261-265. [CrossRef]

18. Huang, Y.-T.; Huang, Y.-H.; Hsieh, C.-H.; Li, C.-J.; Chiu, I.-M. Comparison of Injury Severity Score, Glasgow Coma Scale, and Revised Trauma Score in Predicting the Mortality and Prolonged ICU Stay of Traumatic Young Children: A Cross-Sectional Retrospective Study. Emerg. Med. Int. 2019, 2019, 5453624. [CrossRef]

19. Nogueira, L.S.; de Sousa, R.M.C.; Domingues, C.A. Severity of trauma victims admitted in intensive care units: Comparative study among different indexes. Rev. Lat. Am. Enferm. 2009, 17, 1037-1042. [CrossRef]

20. Cohen, M.J.; Serkova, N.J.; Wiener-Kronish, J.; Pittet, J.F.; Niemann, C.U. 1H-NMR-based metabolic signatures of clinical outcomes in trauma patients-beyond lactate and base deficit. J. Trauma-Inj. Infect. Crit. Care 2010, 69, 31-40. [CrossRef] [PubMed]

21. Yi, L.; Shi, S.; Wang, Y.; Huang, W.; Xia, Z.A.; Xing, Z.; Peng, W.; Wang, Z. Serum Metabolic Profiling Reveals Altered Metabolic Pathways in Patients with Post-traumatic Cognitive Impairments. Sci. Rep. 2016, 6, 21320. [CrossRef]

22. Orešič, M.; Posti, J.P.; Kamstrup-Nielsen, M.H.; Takala, R.S.K.; Lingsma, H.F.; Mattila, I.; Jäntti, S.; Katila, A.J.; Carpenter, K.; Ala-Seppälä, H.; et al. Human Serum Metabolites Associate With Severity and Patient Outcomes in Traumatic Brain Injury. EBioMedicine 2016, 12, 118-126. [CrossRef]

23. Lusczek, E.R.; Muratore, S.L.; Dubick, M.A.; Beilman, G.J. Assessment of key plasma metabolites in combat casualties. J. Trauma Acute Care Surg. 2017, 82, 309-316. [CrossRef]

24. Servia, L.; Jove, M.; Sol, J.; Pamplona, R.; Badia, M.; Montserrat, N.; Portero-Otin, M.; Trujillano, J. A prospective pilot study using metabolomics discloses specific fatty acid, catecholamine and tryptophan metabolic pathways as possible predictors for a negative outcome after severe trauma. Scand. J. Trauma Resusc. Emerg. Med. 2019, 27, 56. [CrossRef]

25. Lusczek, E.R.; Myers, C.; Popovsky, K.; Mulier, K.; Beilman, G.; Sawyer, R. Plasma metabolomics pilot study suggests age and sex-based differences in the metabolic response to traumatic injury. Injury 2018, 49, 2178-2185. [CrossRef]

26. Parent, B.A.; Seaton, M.; Sood, R.F.; Gu, H.; Djukovic, D.; Raftery, D.; O'Keefe, G.E. Use of metabolomics to trend recovery and therapy after injury in critically ill trauma patients. JAMA Surg. 2016, 151, e160853. [CrossRef] 
27. Daley, M.; Dekaban, G.; Bartha, R.; Brown, A.; Stewart, T.C.; Doherty, T.; Fischer, L.; Holmes, J.; Menon, R.; Rupar, C.A.; et al. Metabolomics profiling of concussion in adolescent male hockey players: A novel diagnostic method. Metabolomics 2016, 12, 185. [CrossRef]

28. Cyr, A.; Zhong, Y.; Reis, S.E.; Namas, R.A.; Amoscato, A.; Zuckerbraun, B.; Sperry, J.; Zamora, R.; Vodovotz, Y.; Billiar, T.R. Analysis of the Plasma Metabolome after Trauma, Novel Circulating Sphingolipid Signatures, and In-Hospital Outcomes. J. Am. Coll. Surg. 2021, 232, 276-287. [CrossRef]

29. Langley, R.J.; Tsalik, E.L.; Van Velkinburgh, J.C.; Glickman, S.W.; Rice, B.J.; Wang, C.; Chen, B.; Carin, L.; Suarez, A.; Mohney, R.P.; et al. Sepsis: An integrated clinico-metabolomic model improves prediction of death in sepsis. Sci. Transl. Med. 2013, 5. [CrossRef]

30. Foster, M.A.; Taylor, A.E.; Hill, N.E.; Bentley, C.; Bishop, J.; Gilligan, L.C.; Shaheen, F.; Bion, J.; Fallowfield, J.L.; Woods, D.R.; et al. Mapping the Steroid Response to Major Trauma From Injury to Recovery: A Prospective Cohort Study. J. Clin. Endocrinol. Metab. 2020, 105, 925-937. [CrossRef]

31. Gentile, L.F.; Cuenca, A.G.; Efron, P.A.; Ang, D.; Bihorac, A.; McKinley, B.A.; Moldawer, L.L.; Moore, F.A. Persistent inflammation and immunosuppression: A common syndrome and new horizon for surgical intensive care. J. Trauma Acute Care Surg. 2012, 72 , 1491-1501. [CrossRef]

32. Tiziani, S.; Emwas, A.H.; Lodi, A.; Ludwig, C.; Bunce, C.M.; Viant, M.R.; Günther, U.L. Optimised metabolite extraction from blood serum for 1H nuclear magnetic resonance spectroscopy. Anal. Biochem. 2008, 377, 16-23. [CrossRef]

33. Young, S.P.; Kapoor, S.R.; Viant, M.R.; Byrne, J.J.; Filer, A.; Buckley, C.D.; Kitas, G.D.; Raza, K. The impact of inflammation on metabolomic profiles in patients with arthritis. Arthritis Rheum. 2013, 65, 2015-2023. [CrossRef]

34. Kapoor, S.R.; Filer, A.; Fitzpatrick, M.A.; Fisher, B.A.; Taylor, P.C.; Buckley, C.D.; McInnes, I.; Raza, K.; Young, S.P. Metabolic profiling predicts response to anti-tumor necrosis factor $\alpha$ therapy in patients with rheumatoid arthritis. Arthritis Rheum. 2013, 65, 1448-1456. [CrossRef]

35. Ludwig, C.; Günther, U.L. MetaboLab-advanced NMR data processing and analysis for metabolomics. BMC Bioinform. 2011, 12, 366. [CrossRef]

36. Dieterle, F.; Ross, A.; Schlotterbeck, G.; Senn, H. Probabilistic quotient normalisation as robust method to account for dilution of complex biological mixtures. Application in1H NMR metabonomics. Anal. Chem. 2006, 78, 4281-4290. [CrossRef]

37. Parsons, H.M.; Ludwig, C.; Günther, U.L.; Viant, M.R. Improved classification accuracy in 1- and 2-dimensional NMR metabolomics data using the variance stabilising generalised logarithm transformation. BMC Bioinform. 2007, 8, 234. [CrossRef]

38. Leek, J.T.; Johnson, W.E.; Parker, H.S.; Jaffe, A.E.; Storey, J.D. The SVA package for removing batch effects and other unwanted variation in high-throughput experiments. Bioinformatics 2012, 28, 882-883. [CrossRef]

39. Wishart, D.S.; Feunang, Y.D.; Marcu, A.; Guo, A.C.; Liang, K.; Vázquez-Fresno, R.; Sajed, T.; Johnson, D.; Li, C.; Karu, N.; et al HMDB 4.0: The human metabolome database for 2018. Nucleic Acids Res. 2018, 46, D608-D617. [CrossRef]

40. Chong, J.; Soufan, O.; Li, C.; Caraus, I.; Li, S.; Bourque, G.; Wishart, D.S.; Xia, J. MetaboAnalyst 4.0: Towards more transparent and integrative metabolomics analysis. Nucleic Acids Res. 2018, 46, W486-W494. [CrossRef]

41. Monteleone, P.; Monteleone, A.M.; Troisi, J.; Dalle Grave, R.; Corrivetti, G.; Calugi, S.; Scala, G.; Patriciello, G.; Zanetti, A.; Maj, M Metabolomics signatures of acutely ill and short-term weight recovered women with anorexia nervosa. Mol. Psychiatry 2019, 26, 3980-3991. [CrossRef]

42. Orhon, R.; Eren, S.H.; Karadayi, S.; Korkmaz, I.; Coskun, A.; Eren, M.; Katrancioglu, N. Comparison of trauma scores for predicting mortality and morbidity on trauma patients. Turk. J. Trauma Emerg. Surg. 2014, 20, 258-264. [CrossRef]

43. Peltz, E.D.; D’Alessandro, A.; Moore, E.E.; Chin, T.; Silliman, C.C.; Sauaia, A.; Hansen, K.C.; Banerjee, A. Pathologic metabolism: An exploratory study of the plasma metabolome of critical injury. J. Trauma Acute Care Surg. 2015, 78, 742-751. [CrossRef] [PubMed]

44. Horn, D.L.; Bettcher, L.F.; Navarro, S.L.; Pascua, V.; Neto, F.C.; Cuschieri, J.; Raftery, D.; O’Keefe, G.E. Persistent metabolomic alterations characterise chronic critical illness after severe trauma. J. Trauma Acute Care Surg. 2021, 90, 35-45. [CrossRef]

45. D'Alessandro, A.; Moore, H.B.; Moore, E.E.; Reisz, J.A.; Wither, M.J.; Ghasasbyan, A.; Chandler, J.; Silliman, C.C.; Hansen, K.C.; Banerjee, A. Plasma succinate is a predictor of mortality in critically injured patients. J. Trauma Acute Care Surg. 2017, 83, 491-495. [CrossRef]

46. Wilcox, G. Insulin and insulin resistance. Clin. Biochem. Rev. 2005, 26, 19-39.

47. Bessey, P.Q.; Brooks, D.C.; Black, P.R.; Aoki, T.T.; Wilmore, D.W. Epinephrine acutely mediates skeletal muscle insulin resistance. Surgery 1983, 94, 172-179.

48. Desborough, J.P. The stress response to trauma and surgery. Br. J. Anaesth 2000, 85, 109-117. [CrossRef]

49. Mizock, B.A. Alterations in fuel metabolism in critical illness: Hyperglycaemia. Best Pract. Res. Clin. Endocrinol. Metab. 2001, 15, 533-551. [CrossRef]

50. Thorell, A.; Rooyackers, O.; Myrenfors, P.; Soop, M.; Nygren, J.; Ljungqvist, O.H. Intensive insulin treatment in critically ill trauma patients normalises glucose by reducing endogenous glucose production. J. Clin. Endocrinol. Metab. 2004, 89, 5382-5386. [CrossRef]

51. Kushimoto, S.; Akaishi, S.; Sato, T.; Nomura, R.; Fujita, M.; Kudo, D.; Kawazoe, Y.; Yoshida, Y.; Miyagawa, N. Lactate, a useful marker for disease mortality and severity but an unreliable marker of tissue hypoxia/hypoperfusion in critically ill patients. Acute Med. Surg. 2016, 3, 293-297. [CrossRef] [PubMed] 
52. Hernandez, G.; Bellomo, R.; Bakker, J. The ten pitfalls of lactate clearance in sepsis. Instensive Care Med. 2019, 45, 82-85. [CrossRef]

53. Zhou, Y.; Wang, K.; Zeng, J.; Li, W.; Peng, J.; Zhou, Z.; Deng, P.; Sun, M.; Yang, H.; Li, S.; et al. Metabolic dynamics in critically injured patients: A prospective cohort study integrated with 1H NMR metabolomics. Asia Pac. J. Clin. Nutr. 2019, $28,411-418$. [CrossRef]

54. Blaise, B.J.; Gouel-Chéron, A.; Floccard, B.; Monneret, G.; Allaouchiche, B. Metabolic phenotyping of traumatised patients reveals a susceptibility to sepsis. Anal. Chem. 2013, 85, 10850-10855. [CrossRef]

55. Barton, D.J.; Kumar, R.G.; McCullough, E.H.; Galang, G.; Arenth, P.M.; Berga, S.L.; Wagner, A.K. Persistent hypogonadotropic hypogonadism in men after severe traumatic brain injury: Temporal hormone profiles and outcome prediction. J. Head Trauma Rehabil. 2016, 31, 277-287. [CrossRef] [PubMed]

56. Young, T.P.; Hoaglin, H.M.; Burke, D.T. The role of serum testosterone and TBI in the in-patient rehabilitation setting. Brain Inj. 2007, 21, 645-649. [CrossRef]

57. Almoosa, K.F.; Gupta, A.; Pedroza, C.; Watts, N.B. Low testosterone levels are frequent in patients with acute respiratory failure and are associated with poor outcomes. Endocr. Pract. 2014, 20, 1057-1063. [CrossRef] [PubMed]

58. Rastrelli, G.; Di Stasi, V.; Inglese, F.; Beccaria, M.; Garuti, M.; Di Costanzo, D.; Spreafico, F.; Greco, G.F.; Cervi, G.; Pecoriello, A.; et al. Low testosterone levels predict clinical adverse outcomes in SARS-CoV-2 pneumonia patients. Andrology 2021, 9, 88-98. [CrossRef]

59. Al-Tarrah, K.; Moiemen, N.; Lord, J. The influence of sex steroid hormones on the response to trauma and burn injury. Burn. Trauma 2017, 5, 29. [CrossRef]

60. Preiser, J.C.; Ichai, C.; Orban, J.C.; Groeneveld, A.B.J. Metabolic response to the stress of critical illness. Br. J. Anaesth 2014, 113, 945-954. [CrossRef]

61. Li, H.; Guo, Y.; Yang, Z.; Roy, M.; Guo, Q. The efficacy and safety of oxandrolone treatment for patients with severe burns: A systematic review and meta-analysis. Burns 2016, 42, 717-727. [CrossRef]

62. Hausmann, D.F.; Nutz, V.; Rommelsheim, K.; Caspari, R.; Mosebach, K.O. Anabolic Steroids in Polytrauma Patients. Influence on Renal Nitrogen and Amino Acid Losses: A Double-Blind Study. J. Parenter Enter. Nutr. 1990, 14, 111-114. [CrossRef]

63. Mosebach, K.O.; Hausmann, D.; Caspari, R.; Stoeckel, H. Deca-Durabolin®and parenteral nutrition in post-traumatic patients. Acta Endocrinol. Suppl. 1985, 110, 60-69. [CrossRef]

64. Bulger, E.M.; Jurkovich, G.J.; Farver, C.L.; Klotz, P.; Maier, R.V. Oxandrolone does not improve outcome of ventilator dependent surgical patients. Ann. Surg. 2004, 240, 472-480. [CrossRef]

65. White, H.; Venkatesh, B. Clinical review: Ketones and brain injury. Crit. Care 2011, 15, 219. [CrossRef]

66. Stubbs, B.J.; Koutnik, A.P.; Volek, J.S.; Newman, J.C. From bedside to battlefield: Intersection of ketone body mechanisms in geroscience with military resilience. GeroScience 2020, 43, 1071-1081. [CrossRef]

67. Bradshaw, P.C.; Seeds, W.A.; Miller, A.C.; Mahajan, V.R.; Curtis, W.M. COVID-19: Proposing a Ketone-Based Metabolic Therapy as a Treatment to Blunt the Cytokine Storm. Oxid. Med. Cell Longev. 2020, 2020, 6401341. [CrossRef]

68. Rinehart, D.; Johnson, C.H.; Nguyen, T.; Ivanisevic, J.; Benton, H.P.; Lloyd, J.; Arkin, A.; Deutschbauer, A.M.; Patti, G.J.; Siuzdak, G. Metabolomic data streaming for biology-dependent data acquisition. Nat. Biotechnol. 2014, 32, 524-527. [CrossRef]

69. Karl, J.P.; Margolis, L.M.; Murphy, N.E.; Carrigan, C.T.; Castellani, J.W.; Madslien, E.H.; Teien, H.; Martini, S.; Montain, S.J.; Pasiakos, S.M. Military training elicits marked increases in plasma metabolomic signatures of energy metabolism, lipolysis, fatty acid oxidation, and ketogenesis. Physiol. Rep. 2017, 5, e13407. [CrossRef] [PubMed]

70. Reijnen, A.; Geuze, E.; Vermetten, E. The effect of deployment to a combat zone on testosterone levels and the association with the development of posttraumatic stress symptoms: A longitudinal prospective Dutch military cohort study. Psychoneuroendocrinology 2015, 51, 525-533. [CrossRef]

71. Warren, H.S.; Elson, C.M.; Hayden, D.L.; Schoenfeld, D.A.; Cobb, J.P.; Maier, R.V.; Moldawer, L.L.; Moore, E.E.; Harbrecht, B.G.; Pelak, K.; et al. A genomic score prognostic of outcome in trauma patients. Mol. Med. 2009, 15, 220-227. [CrossRef] [PubMed]

72. Cuenca, A.G.; Gentile, L.F.; Lopez, M.C.; Ungaro, R.; Liu, H.; Xiao, W.; Seok, J.; Mindrinos, M.N.; Ang, D.; Baslanti, T.O.; et al. Development of a genomic metric that can be rapidly used to predict clinical outcome in severely injured trauma patients. Crit. Care Med. 2013, 41, 1175-1185. [CrossRef] [PubMed] 